\title{
The quantum decomposition of random variables without moments
}

\author{
LUIGI ACCARDI \\ Centro Vito Volterra, Facultà di Economia \\ Università di Tor Vergata \\ Via di Tor Vergata, 00133 Roma, Italy \\ accardi@volterra.mat.uniroma2.it \\ HABIB REBEI \\ Department of Mathematics, Higher Institute of Applied Science \\ and Technology of Gabes, University of Gabes, Tunisia \\ habib.rebei@ipein.rnu.tn \\ ANIS RIAHI \\ Department of Mathematics, Higher School of Science \\ and Technology of Hammam Sousse, University of Sousse, Tunisia \\ a1riahi@yahoo.fr \\ Received (Day Month Year) \\ Revised (Day Month Year) \\ Communicated by ( $x x x \times x x x x x x)$
}

The quantum decomposition of a classical random variable is one of the deep results of quantum probability: it shows that any classical random variable or stochastic process has a built in non commutative structure which is intrinsic and canonical, and not artificially put by hands.

Up to now the technique to deduce the quantum decomposition has been based on the theory of interacting Fock spaces and on Jacobi's tri-diagonal relation for orthogonal polynomials. Therefore it requires the existence of moments of any order and cannot be applied to random variables without this property.

The problem to find an analogue of the quantum decomposition for random variables without finite moments of any order remained open for about fifteen years and nobody had any idea of how such a decomposition could look like.

In the present paper we prove that any infinitely divisible random variable has a quantum decomposition canonically associated to its Lévy-Khintchin triple. The analytical formulation of this result is based on Kolmogorov representation of these triples in terms of 1-cocycles (helices) in Hilbert spaces and on the Araki-Woods-Parthasarathy-Schmidt characterization of these representation in terms of Fock spaces. It distinguishes three classes of random variables: (i) with finite second moment; (ii) with finite first moment only; (iii) without any moment, The third class involves a new type of renormalization based on the associated Lévy-Khinchin function.

Keywords: positive definite kernel, Kolmogorov decomposition, Lévy processes, ArakiWoods-Parthasarathy-Schmidt theorem, generalized field operator

AMS Subject Classification: primary 60J65; secondary 60J45, $60 \mathrm{H} 40$. 


\section{Introduction}

This paper is organized as follows:

In Section 2 we recall some known facts about positive definite kernels, their Kolmogorov decomposition and the Araki-Woods-Parthasarathy-Schmidt theorem.

In section 3 we recall the Lévy-Khintchin formula and its connection with positive definite and conditionally positive definite kernels. Then, for any infinitely divisible probability measure $\mu$ on $\mathbb{R}$ with Lévy triple $\left(\alpha, \sigma^{2}, \nu\right)$, we give a concrete realization of the Araki-Woods-Parthasarathy-Schmidt theorem (see ${ }^{22}$ ) by constructing a canonical isomorphism $U$ between $L_{\mathbb{C}}^{2}(\mathbb{R}, \mu)$ and the Boson Fock space $\Gamma\left(\mathbb{C} \oplus L^{2}(\mathbb{R}, \nu)\right)$.

It is known that the random variable with distribution $\mu$ can be identified, up to stochastic equivalence, with the the position operator $q$ on $L_{\mathbb{C}}^{2}(\mathbb{R}, \mu)$. Therefore the canonical isomorphism $U$ induces an action of this operator on the Fock space $\Gamma\left(\mathbb{C} \oplus L^{2}(\mathbb{R}, \nu)\right)$, In Section 4 we prove that this action is implemented by a generalized field operator in the sense of the paper ${ }^{12}$, i.e. the self-adjoint generators of the group generated by the Fock second quantization of translations and unitaries in the one-particle space.

Since the structure of these generators is well known, this gives a natural candidate for the quantum decomposition.

The precise realization of this decomposition however requires some care due to the possible presence of unbounded operators. For example we prove that the vacuum vector is in the domain of the generalized field operator if and only if the second moment of the measure $\mu$ is finite. In such case all coherent vectors are in this domain. Otherwise no coherent vector is in this domain. These equivalent conditions naturally single out a first class of infinitely divisible random variables.

Then we notice that, in the quantum decomposition of these random variables, their first moments explicitly appear. In other words: the finiteness of the first moment is a necessary condition for this form of quantum decomposition. This suggests to introduce a second class of of infinitely divisible random variables: those for which only the first moment is finite. In section (5) we prove that the random variables in this class admit a quantum decomposition which is formally identical to that of the random variables in the first class, but takes place in a weaker topology.

The crucial remark to deal with the third class, consisting of those infinitely divisible random variables with no finite moment, is that the 1-parameter unitary group generated by the generalized field operator can be split as a product of a 1-parameter scalar family times a projective, non unitary representation of the group $\mathbb{R}$ on the Fock space whose multiplier is the exponential of the same additive 2-coboundary which appears in the Araki-Woods-Parthasarathy-Schmidt representation theorem. This projective non unitary representation is differentiable on a 
The quantum decomposition of random variables without moments 3

natural domain and its generator gives the quantum decomposition of the generalized field operator associated to the third class of random variables. It turns out that the quantum decomposition of the random variables in this class coincides with that of the other two classes except for the fact that the constant term is absent. Thus the multiplicative renormalization corresponds, at infinitesimal level, to a formal substraction of the infinite constant corresponding to the first moment.

\section{Preliminaries}

Recall that, given a set $S$, a function $k:(x, y) \in S \times S \longrightarrow k(x, y) \in \mathbb{C}$ is called a positive definite (PD) $\mathbb{C}$-valued kernel if, for every finite subset $F \subseteq S$ the complex square matrix

$$
k(i, j)=: k_{i j}, \quad i, j \in F
$$

is positive definite, i.e. if $\forall d \in \mathbb{N}, \forall x_{1}, \cdots, x_{d} \in S, \lambda_{1}, \cdots, \lambda_{d} \in \mathbb{C}$, one has

$$
\overline{\lambda_{i}} k_{i j} \lambda_{j}:=\sum_{i, j=1}^{d} \bar{\lambda}_{i} k\left(x_{i}, x_{j}\right) \lambda_{j} \geq 0
$$

$k$ is called conditionally positive definite if (2.1) holds whenever the $\lambda_{j}$ 's satisfy the additional condition

$$
\sum_{j=1}^{d} \lambda_{j}=0
$$

For a kernel $q$ on $S$ and for any $x_{0} \in S$ the following statements are equivalent:

(1) $q$ is conditionally positive definite and $q\left(x_{0}, x_{0}\right) \leq 0$,

(2) the kernel

$$
k(x, y):=q(x, y)-q\left(x, x_{0}\right)-q\left(x_{0}, y\right)
$$

is positive definite on $S$.

An infinitely divisible kernel $k$ on $S$ is characterized by the property that for each $t \geq 0$ the kernel

$$
k^{t}(x, y):=(k(x, y))^{t}
$$

is positive definite.

According to Kolmogorov representation theorem a $\mathbb{C}$-valued kernel $k$ on a set $S$ is positive definite if and only if there exists an Hilbert space $\mathcal{H}$ and a map

$$
\text { e. : } x \in S \longmapsto e_{x} \in \mathcal{H}
$$

such that the following two conditions are satisfied:

$$
\begin{gathered}
k(x, y)=\left\langle e_{x}, e_{y}\right\rangle_{\mathcal{H}} \quad ; \quad \forall x, y \in S \\
\left\{e_{x}: x \in S\right\} \text { is total in } \mathcal{H}
\end{gathered}
$$


The pair $(\mathcal{H}, e$.$) is unique up to unitary isomorphism and is called the Kolmogorov$ pair associated to the positive definite kernel $k$.

If $\mathcal{H}$ is an Hilbert space with scalar product $\langle\cdot, \cdot\rangle_{\mathcal{H}}$, the exponential kernel

$$
\exp \langle\cdot, \cdot\rangle_{\mathcal{H}}:(x, y) \in \mathcal{H} \times \mathcal{H} \rightarrow \mathbb{C}
$$

is also a positive definite kernel on $\mathcal{H}$.

Definition 2.1. The Kolmogorov pair associated to the positive definite kernel $\exp \langle\cdot, \cdot\rangle_{\mathcal{H}}$ on $\mathcal{H}$ is denoted

$$
(\Gamma(\mathcal{H}), \operatorname{Exp}(\cdot))
$$

and called the exponential space (or Boson Fock space) over $\mathcal{H}$.

The total set

$$
\operatorname{Exp}(\mathcal{H}):=\left\{\operatorname{Exp}(f):=\sum_{n=0}^{+\infty} \frac{f^{\otimes n}}{\sqrt{n !}} \in \Gamma(\mathcal{H}) ; f \in \mathcal{H}\right\}
$$

is called the set of exponential vectors of $\Gamma(\mathcal{H})$.

The characterizing property of the exponential vectors is

$$
\langle\operatorname{Exp}(f), \operatorname{Exp}(g)\rangle=\left\langle e_{f}, e_{g}\right\rangle=e^{\langle f, g\rangle_{\mathcal{H}}} \quad ; \quad \forall f, g \in \mathcal{H}
$$

Clearly any exponential kernel (2.6) is infinitely divisible. The converse statement is the main point of the Araki-Woods-Parthasarathy-Schmidt theorem.

Theorem 2.1. (Araki-Woods-Parthasarathy-Schmidt)

For a kernel $k$ on a set $S$ the following statements are equivalent:

(i) $k$ is infinitely divisible positive definite

(ii) there exists a conditionally positive definite kernel $q_{0}$ such that $k$ has the form

$$
k(f, g)=e^{q_{0}(f, g)} \quad ; \quad f, g \in S
$$

(iii) For any $f_{0} \in S$ the kernel $q$ on $S$, defined by

$$
q(f, g)=q_{0}(f, g)-q_{0}\left(f, f_{0}\right)-q_{0}\left(f_{0}, g\right)
$$

is positive definite and the map

$$
\kappa: f \in S \longmapsto \kappa_{f}:=-q_{0}\left(f_{0}, f\right) \in \mathbb{C}
$$

is such that, denoting $(\mathcal{H}, e$.$) and (\mathcal{K}, u)$ the Kolmogorov decompositions of $k$ and $q$ respectively, then the map

$$
U: \operatorname{Exp}\left(u_{f}\right) \in \Gamma(\mathcal{K}) \longmapsto e^{\kappa_{f}} e_{f} \in \mathcal{H}
$$

extends to a unitary isomorphism between $\mathcal{H}$ and the Fock space $\Gamma(\mathcal{K})$ over $\mathcal{K}$. 


\section{The Kolmogorov isomorphism associated to an infinitely divisible probability measure on $\mathbb{R}$}

In this section we construct the explicit form of the isomorphism (2.9) in the case in which the kernel $k$ is the one associated to an infinitely divisible law on $\mathbb{R}$.

It is known, (see ${ }^{24}$ ), that any infinitely divisible probability measure $\mu$ on $\mathbb{R}$ is canonically associated to a triple $(\alpha, \sigma, \beta)$ such that:

$-\alpha$ is a real constant

$-\beta$ is a positive finite measure on $\mathbb{R}$ with

$$
\sigma^{2}=\beta(\{0\})
$$

- denoting $\widehat{\mu}$ the Fourier transform of $\mu$ and $\Psi$, the function

$$
\Psi(x)=i \alpha x-\frac{\sigma^{2}}{2} x^{2}+\int_{\mathbb{R} \backslash\{0\}}\left(e^{i x t}-1-\frac{i x t}{1+t^{2}}\right) \frac{1+t^{2}}{t^{2}} d \beta(t) \quad ; \quad x \in \mathbb{R}
$$

one has

$$
\widehat{\mu}(x)=: e^{\Psi(x)} \quad ; \quad x \in \mathbb{R}
$$

Conversely given any such a triple $(\alpha, \sigma, \beta)$, there exists an infinitely divisible probability measure on $\mathbb{R}$ whose has the form (3.2) with $\Psi$ given by (3.1).

The function $\Psi$ is called the Lévy-Khintchine function, or the characteristic exponent, of $\mu$ and the triple $(\alpha, \sigma, \beta)$ is called a generating triple for the measure $\mu$. Finally the measure on $\mathbb{R} \backslash\{0\}$

$$
d \nu(t)=\frac{1+t^{2}}{t^{2}} d \beta(t)
$$

is called the Lévy measure of $\mu$.

In the following the measure $\mu$ will be fixed and the corresponding random variable will be denoted $X_{1}$. For any such $\mu$ the kernel on $\mathbb{R}$

$$
q_{0}(x, y):=\Psi(y-x)
$$

is conditionally positive definite and the kernel

$$
k(x, y):=e^{\Psi(y-x)}
$$

is infinitely divisible and positive definite. Moreover, in this case, the Araki-WoodsParthasarathy-Schmidt isomorphism described in theorem (2.1) can be explicitly expressed in terms of the Lévy triple of $\mu$ as shown in the following theorem.

Theorem 3.1. Let $\mu$ be an infinitely divisible measure with generating triple $(\alpha, \sigma, \beta)$. For each $x \in \mathbb{R}$ and for any $b \in \mathbb{C}$ such that

$$
|b|^{2}=\sigma
$$

denote:

(i) $e_{x} \in L^{2}(\mu)$ the trigonometric function associated to $x$

$$
e_{x}(t):=e^{i t x} \quad ; \quad t \in \mathbb{R}
$$


6 L. Accardi, H. Rebeï and A. Riahi

(ii) $f_{x}$ be the function defined by

$$
f_{x}(t):=e^{i x t}-1
$$

and $\mathcal{K}_{0}$ the space:

$$
\mathcal{K}_{0}:=\text { closed linear span of }\left\{f_{x}, x \in \mathbb{R}\right\} \subseteq L^{2}(\nu)
$$

(iii) $u_{x}$ the vector in $\mathbb{C} \oplus \mathcal{K}_{0}$ given by:

$$
u_{x}=b x+f_{x} \in \mathbb{C} \oplus \mathcal{K}_{0}
$$

where $f_{x}$ is defined by (3.6) and $b$ by (3.4).

Then each $f_{x}(x \in \mathbb{R})$ is in $L^{2}(\nu)$ and the unique linear operator $U$ such that $\forall x \in \mathbb{R}$ :

$$
U: e^{\Psi(x)} \operatorname{Exp}\left(u_{x}\right) \in \Gamma\left(\mathbb{C} \oplus \mathcal{K}_{0}\right) \longrightarrow U\left(e^{\Psi(x)} \operatorname{Exp}\left(u_{x}\right)\right):=e_{x} \in L^{2}(\mu)
$$

is a unitary isomorphism from the Fock space $\Gamma\left(\mathbb{C} \oplus \mathcal{K}_{0}\right)$, over $\mathbb{C} \oplus \mathcal{K}_{0}$, to $L^{2}(\mu)$.

Remark 3.1. The linear independence of the $f_{x} \mathrm{~s}(\in \mathbb{R})$ depends on the support of the Lévy measure of $\mu$. However in any case there exists a subset $S_{0} \subseteq \mathbb{R}$ such that $\left\{f_{x}: x \in S_{0}\right\}$ is a linearly independent set and

$$
\mathcal{K}_{0}:=\text { closed linear span of }\left\{f_{x}, x \in S_{0}\right\}
$$

Proof. The fact that the set

$$
\mathcal{E}:=\left\{f_{x} ; x \in \mathbb{R}\right\}
$$

is contained $L^{2}(\nu)$, i.e. that the integral

$$
\int_{\mathbb{R} \backslash\{0\}}\left|f_{x}(t)\right|^{2} \nu(d t)=\int_{\mathbb{R} \backslash\{0\}}\left|f_{x}(t)\right|^{2} \frac{1+t^{2}}{t^{2}} \beta(d t)
$$

is finite for all $x \in \mathbb{R}$,is well known. We include a proof for completeness. Notice that, as $t \longrightarrow 0$

$$
\left|f_{x}(t)\right|^{2} \frac{1+t^{2}}{t^{2}}=2(1-\cos (x t)) \frac{1+t^{2}}{t^{2}} \sim x^{2}
$$

while, as $t \longrightarrow \infty$

$$
\left|f_{x}(t)\right|^{2} \frac{1+t^{2}}{t^{2}} \leq 4 \frac{1+t^{2}}{t^{2}} \sim 4
$$

Therefore the measurable function

$$
t \mapsto\left|f_{x}(t)\right|^{2} \frac{1+t^{2}}{t^{2}}
$$

is bounded hence integrable w.r.t. the finite measure $\beta$.

In the notations of theorem (2.1) we choose:

$$
S=\mathbb{R} \quad ; \quad k(x, y)=e^{\Psi(y-x)} \quad ; \quad q_{0}(x, y)=\Psi(y-x) \quad ; \quad f_{0}:=0 \in \mathbb{R}
$$


Then, using the expression (3.1) for the Lévy-Khintchine function, one has

$$
\begin{aligned}
q_{0}(x, y) & =\Psi(y-x) \\
& =i \alpha(y-x)-\frac{\sigma^{2}}{2}(y-x)^{2}+\int_{\mathbb{R} \backslash\{0\}}\left(e^{i(y-x) t}-1-\frac{i(y-x) t}{1+t^{2}}\right) \nu(d t)
\end{aligned}
$$

Therefore since, in the notation (2.8):

$$
\kappa_{x}=-q_{0}(0, x)=-i \alpha x+\frac{\sigma^{2}}{2} x^{2}-\int_{\mathbb{R} \backslash\{0\}}\left(e^{i x t}-1-\frac{i x t}{1+t^{2}}\right) \nu(d t)
$$

Thus, with the above choices, the kernel $q$ defined by (2.7) is given by:

$$
\begin{aligned}
q(x, y) & =i \alpha(y-x)-\frac{\sigma^{2}}{2}(y-x)^{2}+\int_{\mathbb{R} \backslash\{0\}}\left(e^{i(y-x) t}-1-\frac{i(y-x) t}{1+t^{2}}\right) \nu(d t) \\
& +i \alpha x-\frac{\sigma^{2}}{2} x^{2}+\int_{\mathbb{R} \backslash\{0\}}\left(e^{-i x t}-1+\frac{i x t}{1+t^{2}}\right) \nu(d t) \\
& -i \alpha y-\frac{\sigma^{2}}{2} y^{2}+\int_{\mathbb{R} \backslash\{0\}}\left(e^{i y t}-1-\frac{i y t}{1+t^{2}}\right) \nu(d t) \\
& =\sigma^{2} x y+\int_{\mathbb{R} \backslash\{0\}}\left(e^{i(y-x) t}-e^{-i x t}-e^{i y t}+1\right) \nu(d t) \\
& =\sigma^{2} x y+\int_{\mathbb{R} \backslash\{0\}} \overline{f_{x}(t)} f_{y}(t) \nu(d t)
\end{aligned}
$$

The right hand side of (3.11) suggests a natural choice for a Kolmogorov representation of the kernel $q$. In fact the first term of the sum is a scalar product on $\mathbb{R}$ and the second, due to the linear independence of the $f_{x}$ 's, $x \in S_{0}$ extends to a scalar product on the space $\mathcal{K}_{0}$, defined by (3.7). The complexification of the inner product (3.11) gives a scalar product on the space

$$
\mathcal{K}:=\mathbb{C} \oplus \mathcal{K}_{0}
$$

with inner product

$$
\langle\cdot, \cdot\rangle_{\mathcal{K}}:=\langle\cdot, \cdot\rangle:=\langle\cdot, \cdot\rangle_{\mathbb{C}}+\langle\cdot, \cdot\rangle_{L^{2}(\nu)}
$$

From the definition of $\mathcal{K}_{0}$ it is clear that the range of the map (3.8) is total in $\mathbb{C} \oplus \mathcal{K}_{0}$. Therefore the pair $(\mathcal{K}, u)$ defined respectively by (3.12) and (3.8) is a Kolmogorov representation of the kernel $q$. Passing to the exponential space $\Gamma(\mathcal{K})$ of $\mathcal{K}$ the exponential kernel of the scalar product (3.13) is:

$$
\left\langle\operatorname{Exp}\left(u_{x}\right), \operatorname{Exp}\left(u_{y}\right)\right\rangle=e^{\left\langle u_{x}, u_{y}\right\rangle}=e^{q(x, y)}
$$

On the other hand one has:

$$
e^{\Psi(y-x)}=\int_{\mathbb{R}} e^{-i x t} e^{i y t} \mu(d t)=\hat{\mu}(y-x)=\left\langle e_{x}, e_{y}\right\rangle_{L^{2}(\mu)}
$$


and the family of trigonometric exponentials $\left\{e_{x}, x \in \mathbb{R}\right\}$ is total in $L^{2}(\mu)$. It follows that, if we define the linear map

$$
U: \Gamma(\mathcal{K})=\Gamma\left(\mathbb{C} \oplus L^{2}(\nu)\right) \longrightarrow L^{2}(\mu)
$$

by linear extension of

$$
U\left(e^{\Psi(x)} \operatorname{Exp}\left(u_{x}\right)\right)=e_{x} \quad ; \quad x \in \mathbb{R}
$$

then we get

$$
\begin{gathered}
\left\langle e_{x}, e_{y}\right\rangle_{L^{2}(\mu)}=\widehat{\mu}(y-x)=e^{\Psi(y-x)}=e^{q_{0}(x, y)}=e^{q(x, y)+q_{0}(x, 0)+q_{0}(0, y)}= \\
=e^{\left\langle u_{x}, u_{y}\right\rangle+\overline{\Psi(x)}+\Psi(y)}=\left\langle e^{\Psi(x)} \operatorname{Exp}\left(u_{x}\right), e^{\Psi(y)} \operatorname{Exp}\left(u_{y}\right)\right\rangle_{\Gamma(\mathcal{K})}
\end{gathered}
$$

Therefore the pair

$$
(\mathcal{H}, e):=\left(L^{2}(\mu),\left(e_{x}\right)_{x \in \mathbb{R}}\right)
$$

is a Kolmogorov representation of the kernel $\operatorname{Exp}\langle\cdot, \cdot\rangle_{\mathcal{K}}$.

Example 3.1. (Gaussian case)

Let $X_{1} \sim \mathcal{N}\left(0, \sigma^{2}\right)=: \mu_{G}$ be the mean zero, real valued, Gaussian random variable with variance $\sigma^{2}$. Then

$$
q(x, y)=\sigma^{2} x y \quad ; \quad k(x, y)=\exp \left\{-\frac{\sigma^{2}}{2}|y-x|^{2}\right\} \quad ; \quad-\Psi(x)=\frac{\sigma^{2}}{2} x^{2}
$$

In this case

$$
\mathcal{K}=\mathbb{C} \quad ; \quad \mathcal{H}=L^{2}\left(\mu_{G}\right) \quad ; \quad u_{x}=b x \in \mathbb{C},
$$

with $|b|=\sigma$ and $e_{x}$ given by (3.5). Hence the Gaussian isomorphism is given by

$$
U_{G}: \Gamma(\mathbb{C}) \ni e^{-\frac{\sigma^{2}}{2} x^{2}} \operatorname{Exp}(b x) \longmapsto e_{x} \in L^{2}\left(\mu_{G}\right)
$$

Example 3.2. (Poisson case)

Let $X_{1} \sim \prod(\lambda)=: \mu_{P}$ be the Poisson random variable with intensity $\lambda>0$. In this case

$$
\mathcal{H}=L^{2}\left(\mu_{P}\right)=\left\{z=\left(z_{n}\right)_{n}, \sum_{n=0}^{\infty} \frac{\lambda^{n}}{n !}\left|z_{n}\right|^{2}<\infty\right\} \equiv \ell^{2}(\mathbb{N})
$$

and we refer it to the orthonormal basis:

$$
e_{x}=\left(e^{i n x}\right)_{n}
$$

Introducing the function (extended to zero by continuity)

one has

$$
e_{1}: x \in \mathbb{R} \rightarrow e_{1}(x):=\frac{e^{x}-1}{x} \in \mathbb{R}
$$

$$
q(x, y)=\lambda x y e_{1}(-i x) e_{1}(i y)=\lambda x y \overline{e_{1}(i x)} e_{1}(i y)
$$


The quantum decomposition of random variables without moments 9

$$
\begin{gathered}
k(x, y)=\exp \left\{i \lambda(y-x) e_{1}(i(y-x))\right\} \quad ; \quad-\Psi(x)=-i \lambda x e_{1}(i x) . \\
\mathcal{K}=\mathbb{C} \quad ; \quad u_{x}=\sqrt{\lambda} x e_{1}(i x) \in \mathbb{C}
\end{gathered}
$$

As a consequence we obtain the following Poisson isomorphism

$$
U_{P}: \Gamma(\mathbb{C}) \ni e^{\lambda\left(e^{i x}-1\right)} \operatorname{Exp}\left(\sqrt{\lambda} x e_{1}(i x)\right) \longmapsto\left(e^{i n x}\right)_{n} \in L^{2}\left(\mu_{P}\right)
$$

Example 3.3. (Gamma case)

Let $X_{1} \sim \Gamma(\alpha)$ be the Gamma random variable with parameter $\alpha>0$. Then

$$
q(x, y)=\int_{\mathbb{R}}\left(e^{-i x t}-1\right)\left(e^{i y t}-1\right) \nu_{\Gamma}(d t)
$$

where

$$
\nu_{\Gamma}(d t)=\frac{e^{-\alpha t}}{t} 1_{(0, \infty)}
$$

is the Lévy measure of the Gamma distribution $\mu_{\Gamma}$. By a simple calculation we have

$$
k(x, y)=e^{-\alpha \log (1-i(y-x))} \quad ; \quad-\Psi(x)=\alpha \log (1-i x)
$$

In this case

$$
\mathcal{K}=L^{2}\left(\nu_{\Gamma}\right) \quad ; \quad \mathcal{H}=L^{2}\left(\mu_{\Gamma}\right) \quad ; \quad u_{x}=f_{x},
$$

where $f_{x}$ is given by Eq. (3.6) and we obtain the Gamma isomorphism

$$
U_{\Gamma}: \Gamma\left(L^{2}\left(\nu_{\Gamma}\right)\right) \ni e^{-\alpha \log (1-i x)} \operatorname{Exp}\left(e_{x}-1\right) \longmapsto e_{x} \in L^{2}\left(\mu_{\Gamma}\right)
$$

Lemma 3.1. If the Lévy measure $\nu$ (see (3.3)) has finite second order moment

$$
\int_{\mathbb{R} \backslash\{0\}}|t|^{2} \nu(d t)<+\infty
$$

then $\mathcal{K}_{0}=L^{2}(\nu)$.

Proof. We know from Theorem (3.1) that $\mathcal{E}$ is a subset of $L^{2}(\nu)$. Let $f \in L^{2}(\nu)$ satisfy

$$
\left\langle f_{x}, f\right\rangle=\int_{\mathbb{R} \backslash\{0\}} \overline{f_{x}(t)} f(t) \nu(d t)=0 \quad ; \quad \forall x \in \mathbb{R}
$$

and consider the function

$$
F(x):=\int_{\mathbb{R} \backslash\{0\}} h(x, t) \nu(d t)
$$

where $h(t, x)=\left(e^{i x t}-1\right) f(t)$. To prove that $F$ is derivable it is sufficient to check the two following conditions.

(i) $x \longmapsto h(x, t)$ is derivable on $\mathbb{R}$ for $\nu$-a.e. $t \in \mathbb{R} \backslash\{0\}$.

(ii) $\frac{\partial}{\partial x} h(x, t)$ exists on $\mathbb{R}$ for $\nu$-a.e. $t \in \mathbb{R} \backslash\{0\}$ and $\left|\frac{\partial}{\partial x} h(x, t)\right|$ is dominated by a $\nu$-integrable function $\varphi(t)$, independent of $x$. 
Condition $(i)$ is easily checked and we have

$$
\left|\frac{\partial}{\partial x} h(x, t)\right|=\left|i t e^{i x t} f(t)\right|=|t f(t)|=: \varphi(t)
$$

But

$$
\int_{\mathbb{R} \backslash\{0\}} \varphi(t) \nu(d t) \leq\left(\int_{\mathbb{R} \backslash\{0\}} t^{2} \nu(d t)\right)^{\frac{1}{2}}\left(\int_{\mathbb{R} \backslash\{0\}}|f(t)|^{2} \nu(d t)\right)^{\frac{1}{2}}<+\infty
$$

This gives (ii). Then from (3.15), we deduce that $F(x)=0 \forall x \in \mathbb{R}$. Hence

$$
F^{\prime}(x)=\int_{\mathbb{R} \backslash\{0\}} i t e^{i x t} f(t) \nu(d t)=0 \quad \forall x \in \mathbb{R}
$$

which is equivalent to $\hat{\rho}(x)=0$, where the the signed measure $\rho$ is given by

$$
\rho(d t)=t f(t) \nu(d t)
$$

This gives that $\rho$ is the null measure which implies that $f=0$.

\section{The generalized field operator}

It is known that the bosonic Fock space $\Gamma(\mathcal{H})$ can be represented in the form

$$
\Gamma(\mathcal{H}):=\bigoplus_{n=0}^{+\infty} \mathcal{H}^{\circ n}
$$

where $\mathcal{H}^{\circ n}$ denotes the $n$-th symmetric tensor power of $\mathcal{H}$ and by definition $\mathcal{H}^{\circ n}:=$ $\mathbb{C} \Phi$ where $\Phi$ is the vacuum vector. Using this identification the bosonic creation and annihilation operators are defined, on the total set

$$
\left\{v_{1} \circ \cdots \circ v_{n} \in \mathcal{H}^{\circ n}: v_{1}, \ldots, v_{n} \in \mathcal{H}\right\}
$$

as follows: for $u \in \mathcal{H}$,

$$
\begin{gathered}
A^{+}(u): v_{1} \circ \cdots \circ v_{n} \in \mathcal{H}^{\circ n} \longmapsto \sqrt{n+1} u \circ v_{1} \circ \cdots \circ v_{n} \in \mathcal{H}^{\circ(n+1)} \\
A^{+}(u) \Phi=u \\
A^{-}(u): v_{1} \circ \cdots \circ v_{n} \in \mathcal{H}^{\circ n} \longmapsto \frac{1}{\sqrt{n}} \sum_{i=1}^{n}\left\langle u, v_{i}\right\rangle v_{1} \circ \cdots \circ{\widehat{v_{i}}}^{\circ} \circ \cdots v_{n} \in \mathcal{H}^{\circ(n-1)} \\
A^{-}(u) \Phi=0,
\end{gathered}
$$

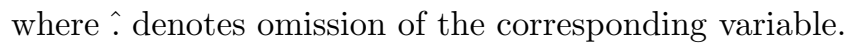

Definition 4.1. The differential second quantized $\Lambda(T)$ of a self-adjoint operator $T$ of $\mathcal{H}$ is defined via the Stone theorem by

$$
\Gamma\left(e^{i t T}\right)=: e^{i t \Lambda(T)} \quad, \quad t \in \mathbb{R},
$$

where for an unitary operator $X, \Gamma(X)$ is the second quantized of $X$. 
The creation, annihilation operators and second quantized operator of $T$ act on the domain of the exponential vectors as follows:

$$
A^{-}(u) \operatorname{Exp}(x):=\langle u, x\rangle \operatorname{Exp}(x) \quad, \quad A^{+}(u) \operatorname{Exp}(x):=\left.\frac{d}{d s}\right|_{s=0} \operatorname{Exp}(x+s u)
$$

and

$$
\Gamma(T) \operatorname{Exp}(x):=\operatorname{Exp}(T x)
$$

It follows that if $x \in \operatorname{Dom}(T)$

$$
\Lambda(T) \operatorname{Exp}(x)=-\left.i \frac{d}{d s}\right|_{s=0} \operatorname{Exp}\left(e^{i s T} x\right)=A^{+}(T x) \operatorname{Exp}(x)
$$

Definition 4.2. Let $q$ be the multiplication (position) operator in $L^{2}(\mu)$ :

$$
(q f)(t):=t f(t) \quad ; \quad f \in L^{2}(\mu), t \in \mathbb{R}
$$

Define the operator $Q$ on $\Gamma\left(\mathbb{C} \oplus \mathcal{K}_{0}\right)$ by

$$
Q:=U^{*} q U
$$

where $U$ is the isomorphism defined by (3.9). Since $\mu$ is a finite measure on $\mathbb{R}, q$ is self-adjoint (see ${ }^{23}$ Proposition 1, chapter VIII. 3) and

$$
e^{i t Q}=U^{*} e^{i t q} U \quad, \quad t \in \mathbb{R}
$$

Moreover $Q$ is called the generalized field operator.

Lemma 4.1. The one-parameter unitary group

$$
t \mapsto e^{i t Q}
$$

acts on the total set $\left\{\operatorname{Exp}\left(u_{x}\right), x \in \mathbb{R}\right\}$ as follows:

$$
e^{i t Q} \operatorname{Exp}\left(u_{x}\right)=e^{\Psi(x+t)-\Psi(x)} \operatorname{Exp}\left(u_{x+t}\right)
$$

Proof. From the definition (3.9) of the isomorphism $U$ we get

$$
\begin{aligned}
e^{i t Q} \operatorname{Exp}\left(u_{x}\right) & =U^{*} e^{i t q} U \operatorname{Exp}\left(u_{x}\right)=U^{*} e^{i t q}\left(e^{-\Psi(x)} e_{x}\right)=e^{-\Psi(x)} U^{*}\left(e^{i t q} e^{i x(\cdot)}\right) \\
& =e^{-\Psi(x)} U^{*}\left(e^{i(t+x)(\cdot)}\right)=e^{-\Psi(x)} U^{*}\left(e_{x+t}\right) \\
& =e^{-\Psi(x)} e^{\Psi(x+t)} U^{*}\left(e^{-\Psi(x+t)} e_{x+t}\right) \\
& =e^{-\Psi(x)} e^{\Psi(x+t)} \operatorname{Exp}\left(u_{x+t}\right)=e^{\Psi(x+t)-\Psi(x)} \operatorname{Exp}\left(u_{x+t}\right)
\end{aligned}
$$

Lemma 4.2. The following statements are equivalent:

(i) The second moment of $\mu$ is finite. 
(ii) The vacuum vector is in the domain $\mathcal{D}(Q)$ of $Q$.

(iii) There exists $y \in \mathbb{R}$ such that $\operatorname{Exp}\left(u_{y}\right)$ is in the domain $\mathcal{D}(Q)$ of $Q$.

(iv) The total set $\left\{\operatorname{Exp}\left(u_{y}\right), y \in \mathbb{R}\right\}$ is in the domain of $Q$.

Proof. The domain $\mathcal{D}(q)$ of the multiplication operator $q$ is defined by

$$
\mathcal{D}(q):=\left\{f \in L^{2}(\mu) \quad, \quad x f \in L^{2}(\mu)\right\}
$$

Therefore, given $y \in \mathbb{R}, \operatorname{Exp}\left(u_{y}\right) \in \mathcal{D}(Q)$ if and only if

$$
\begin{aligned}
+\infty>\left\|Q\left(\operatorname{Exp}\left(u_{y}\right)\right)\right\|^{2} & =\left\|U^{*} Q U\left(\operatorname{Exp}\left(u_{y}\right)\right)\right\|^{2}=\left\|q\left(e^{-\Psi(y)} e_{y}\right)\right\|^{2} \\
& =e^{-2 \Re(\Psi(y))}\left\langle e_{y}, q^{2} e_{y}\right\rangle=e^{-2 \Re(\Psi(y))} \int_{\mathbb{R} \backslash\{0\}} t^{2} \mu(d t) \\
& =e^{-2 \Re(\Psi(y))}\left\langle\Phi, Q^{2} \Phi\right\rangle_{\Gamma(\mathcal{K})}
\end{aligned}
$$

From which the thesis immediately follows.

Proposition 4.1. If the second moment of $\mu$ is finite, the generalized field operator $Q$ acts on the total set $\left\{\operatorname{Exp}\left(u_{x}\right), x \in \mathbb{R}\right\}$ as follows:

$$
Q\left(\operatorname{Exp}\left(u_{x}\right)\right)=\left(A^{+}\left(h_{x}\right)+A^{-}\left(h_{x}\right)+\lambda(x)\right) \operatorname{Exp}\left(u_{x}\right)
$$

where

$$
h_{x}:=-i b+q e_{x} \quad ; \quad \lambda(x)=\mathbb{E}\left(X_{1}\right)-2 \Re\left\langle h_{x}, u_{x}\right\rangle
$$

where $\mathbb{E}\left(X_{1}\right)$ denotes the first moment of $\mu$ (which under our assumptions exists).

Proof. It is known (see ${ }^{24}$ ) that $\mu$ has a finite second moment if and only if $\nu$ has the same property and in this case $\Psi$ is twice differentiable. Thus one can take the derivative at $t=0$ of equation (4.6) obtaining

$$
i Q \operatorname{Exp}\left(u_{x}\right)=\Psi^{\prime}(x) \operatorname{Exp}\left(u_{x}\right)+\left.\frac{d}{d t}\right|_{t=0} \operatorname{Exp}\left(u_{x+t}\right)
$$


But with notation $f(t)=u_{x+t}-u_{x}$ one has

$$
\begin{aligned}
\left.\frac{d}{d t}\right|_{t=0} \operatorname{Exp}\left(u_{x+t}\right) & =\lim _{t \rightarrow 0} \sum_{n=0}^{+\infty} \frac{1}{\sqrt{n !}} \frac{\left(u_{x+t}\right)^{\otimes n}-\left(u_{x}\right)^{\otimes n}}{t} \\
& =\sum_{n=1}^{+\infty} \frac{1}{\sqrt{n !}} \lim _{t \rightarrow 0} \frac{\left(u_{x+t}\right)^{\otimes n}-\left(u_{x}\right)^{\otimes n}}{t} \\
& =\sum_{n=1}^{+\infty} \frac{1}{\sqrt{n !}} \lim _{t \rightarrow 0} \frac{\left(f(t)+u_{x}\right)^{\otimes n}-\left(u_{x}\right)^{\otimes n}}{t} \\
& =\sum_{n=1}^{+\infty} \frac{1}{\sqrt{n !}} \lim _{t \rightarrow 0} \sum_{k=1}^{n}\left(\begin{array}{l}
n \\
k
\end{array}\right) \frac{f(t)}{t} \widehat{\otimes}(f(t))^{\widehat{\otimes}(k-1)} \widehat{\otimes}\left(u_{x}\right)^{\widehat{\otimes}(n-k)} \\
& =\sum_{n=1}^{+\infty} \frac{\sqrt{n}}{\sqrt{(n-1) !}} f^{\prime}(0) \widehat{\otimes}\left(u_{x}\right)^{\widehat{\otimes}(n-1)}
\end{aligned}
$$

Note that

$$
\begin{aligned}
f^{\prime}(0) & =\lim _{t \rightarrow 0} \frac{u_{x+t}-u_{x}}{t} \\
& =\lim _{t \rightarrow 0} \frac{b(x+t)-b x}{t}+\frac{f_{x+t}-f_{x}}{t} \\
& =b+i q e_{x} \\
& =i h_{x}
\end{aligned}
$$

Then from (4.1) we conclude that

$$
\left.\frac{d}{d t}\right|_{t=0} \operatorname{Exp}\left(u_{x+t}\right)=\sum_{n=1}^{+\infty} \frac{\sqrt{n}}{\sqrt{(n-1) !}} i h_{x} \widehat{\otimes}\left(u_{x}\right)^{\otimes(n-1)}=i A^{+}\left(h_{x}\right) \operatorname{Exp}\left(u_{x}\right) .
$$

On the other hand we have

$$
\begin{aligned}
\Psi^{\prime}(x) & =i \alpha-\sigma^{2} x+\int_{\mathbb{R} \backslash\{0\}}\left(i t e^{i x t}-\frac{i t}{1+t^{2}}\right) \nu(d t) \\
& =i \alpha-\sigma^{2} x+\int_{\mathbb{R} \backslash\{0\}}\left(i t e^{i x t}-i t\right) \nu(d t)+\int_{\mathbb{R} \backslash\{0\}}\left(i t-\frac{i t}{1+t^{2}}\right) \nu(d t) \\
& =i \alpha-\sigma^{2} x+i \int_{\mathbb{R} \backslash\{0\}}\left(t e^{i x t}-t\right) \nu(d t)+i \int_{\mathbb{R} \backslash\{0\}} t \beta(d t)
\end{aligned}
$$

and

$$
\begin{aligned}
\left\langle u_{x}, h_{x}\right\rangle & =i \sigma^{2} x+\int_{\mathbb{R} \backslash\{0\}} \overline{\left(e^{i x t}-1\right)} t e^{i x t} \nu(d t) \\
& =i \sigma^{2} x+\int_{\mathbb{R} \backslash\{0\}}\left(t-t e^{i x t}\right) \nu(d t) .
\end{aligned}
$$

Then using the fact that

$$
\mathbb{E}\left(X_{1}\right)=-i \Psi^{\prime}(0)=\alpha+\int_{\mathbb{R} \backslash\{0\}} t \beta(d t),
$$


we get

$$
\Psi^{\prime}(x)=i \mathbb{E}\left(X_{1}\right)-i\left\langle u_{x}, h_{x}\right\rangle
$$

Thus

$$
\Psi^{\prime}(x)=i\left(\mathbb{E}\left(X_{1}\right)-2 \Re\left(\left\langle h_{x}, u_{x}\right\rangle\right)\right)+i\left\langle h_{x}, u_{x}\right\rangle=i \lambda(x)+i\left\langle h_{x}, u_{x}\right\rangle
$$

and we obtain

$$
\begin{aligned}
Q \operatorname{Exp}\left(u_{x}\right) & =\lambda(x) \operatorname{Exp}\left(u_{x}\right)+\left\langle h_{x}, u_{x}\right\rangle \operatorname{Exp}\left(u_{x}\right)+A^{+}\left(h_{x}\right) \operatorname{Exp}\left(u_{x}\right) \\
& =A^{+}\left(h_{x}\right) \operatorname{Exp}\left(u_{x}\right)+A^{-}\left(h_{x}\right) \operatorname{Exp}\left(u_{x}\right)+\lambda(x) \operatorname{Exp}\left(u_{x}\right) .
\end{aligned}
$$

Theorem 4.1. Assume that the second moment of $\mu$ is finite. Then under the identification

$$
\begin{gathered}
\Gamma\left(\mathbb{C} \oplus L^{2}(\nu)\right) \equiv \Gamma(\mathbb{C}) \otimes \Gamma\left(L^{2}(\nu)\right)=: \Gamma_{G} \otimes \Gamma_{C P} \\
\operatorname{Exp}(z \oplus f) \equiv \operatorname{Exp}(z) \otimes \operatorname{Exp}(f),
\end{gathered}
$$

the generalized field operator $Q$ takes the form

$$
Q=Q_{G} \otimes 1+1 \otimes Q_{C P}
$$

where

$$
\begin{array}{r}
Q_{G}=A^{+}(-i b)+A^{-}(-i b)=i\left(\bar{b} a-b a^{+}\right) \\
Q_{C P}=A_{\nu}^{+}(q \cdot 1)+A_{\nu}^{-}(q \cdot 1)+\Lambda_{\nu}(q)+\mathbb{E}\left(X_{1}\right) \mathbf{1}
\end{array}
$$

and $a, a^{+}$are the usual $1-$ mode creation and annihilation operators and $A_{\nu}^{+}, A_{\nu}^{-}, \Lambda_{\nu}$ are the creation, annihilation and preservation operators in the Fock representation of $L^{2}(\nu)$.

Remark 4.1. Notice that in general the constant function $1 \notin L^{2}(\nu)$. However, if the second moment of $\mu$ is finite, then $q \cdot 1 \in L^{2}(\nu)$ so that expressions like $A_{\nu}^{ \pm}(q \cdot 1)$ make sense. 
Proof. By using Proposition (4.1) and the identification (4.8), we have

$$
\begin{aligned}
Q \operatorname{Exp}\left(u_{x}\right) & =\left.\frac{d}{d s}\right|_{s=0} \operatorname{Exp}\left(u_{x}+s h_{x}\right)+\left\langle-i b+q e_{x}, b x+f_{x}\right\rangle \operatorname{Exp}\left(b x+f_{x}\right) \\
& +\left(\mathbb{E}\left(X_{1}\right)-2 \Re\left(\left\langle h_{x}, u_{x}\right\rangle\right)\right) \operatorname{Exp}\left(b x+f_{x}\right) \\
& =\left.\frac{d}{d s}\right|_{s=0} \operatorname{Exp}\left((b x+s(-i b))+\left(f_{x}+s q e_{x}\right)\right) \\
& +\left(\langle-i b, b x\rangle+\left\langle q e_{x}, f_{x}\right\rangle\right) \operatorname{Exp}\left(b x+f_{x}\right) \\
& +\left(\mathbb{E}\left(X_{1}\right)-2 \Re\left(\left\langle h_{x}, u_{x}\right\rangle\right)\right) \operatorname{Exp}\left(b x+f_{x}\right) \\
& =\left.\frac{d}{d s}\right|_{s=0}\left(\operatorname{Exp}(b x+s(-i b)) \otimes \operatorname{Exp}\left(f_{x}+s q e_{x}\right)\right) \\
& +\left(\langle-i b, b x\rangle+\left\langle q e_{x}, f_{x}\right\rangle\right) \operatorname{Exp}\left(b x+f_{x}\right) \\
& +\left(\mathbb{E}\left(X_{1}\right)-2 \Re\left(\left\langle h_{x}, u_{x}\right\rangle\right)\right) \operatorname{Exp}\left(b x+f_{x}\right) \\
& \left.=\left(\left.\frac{d}{d s}\right|_{s=0} \operatorname{Exp}(b x+s(-i b))\right) \otimes \operatorname{Exp}_{f}\right) \\
& +\operatorname{Exp}(b x) \otimes\left(\left.\frac{d}{d s}\right|_{s=0} \operatorname{Exp}\left(f_{x}+\operatorname{sqe} e_{x}\right)\right) \\
& +\langle-i b, b x\rangle \operatorname{Exp}(b x) \otimes \operatorname{Exp}\left(f_{x}\right)+\left\langle q e_{x}, f_{x}\right\rangle \operatorname{Exp}(b x) \otimes \operatorname{Exp}\left(f_{x}\right) \\
& \left(\mathbb{E}\left(X_{1}\right)-2 \Re\left(\left\langle h_{x}, u_{x}\right\rangle\right)\right) \operatorname{Exp}(b x) \otimes \operatorname{Exp}\left(f_{x}\right)
\end{aligned}
$$

Hence by equation (4.4) we have

$$
\begin{aligned}
Q \operatorname{Exp}\left(u_{x}\right) & =\left(A^{+}(-i b) \operatorname{Exp}(b x)\right) \otimes \operatorname{Exp}\left(f_{x}\right)+\operatorname{Exp}(b x) \otimes\left(A_{\nu}^{+}\left(q e_{x}\right) \operatorname{Exp}\left(f_{x}\right)\right) \\
& +\left(A^{-}(-i b) \operatorname{Exp}(b x)\right) \otimes \operatorname{Exp}\left(f_{x}\right)+\operatorname{Exp}(b x) \otimes\left(A_{\nu}^{-}\left(q e_{x}\right) \operatorname{Exp}\left(f_{x}\right)\right) \\
& +\operatorname{Exp}(b x) \otimes\left(\lambda(x) \operatorname{Exp}\left(f_{x}\right)\right) \\
& =\left[\left(A^{+}(-i b)+A^{-}(-i b)\right) \operatorname{Exp}(b x)\right) \otimes \operatorname{Exp}\left(f_{x}\right) \\
& +\operatorname{Exp}(b x) \otimes\left[\left(A_{\nu}^{+}\left(q e_{x}\right)+A_{\nu}^{-}\left(q e_{x}\right)+\lambda(x)\right) \operatorname{Exp}\left(f_{x}\right)\right],
\end{aligned}
$$


where

$$
\begin{aligned}
\lambda(x) & =-2 \int_{\mathbb{R} \backslash\{0\}}(t-t \cos (x t)) \nu(d t)+\mathbb{E}\left(X_{1}\right) \\
& =\int_{\mathbb{R} \backslash\{0\}}\left[-2 t+t\left(e^{i x t}+e^{-i x t}\right)\right] \nu(d t)+\mathbb{E}\left(X_{1}\right) \\
& =\int_{\mathbb{R} \backslash\{0\}}\left(-t+t e^{i x t}\right) \nu(d t)+\int_{\mathbb{R} \backslash\{0\}}\left(-t+t e^{-i x t}\right) \nu(d t)+\mathbb{E}\left(X_{1}\right) \\
& =-\left(\left\langle q e_{x}, f_{x}\right\rangle+\left\langle f_{x}, q e_{x}\right\rangle\right)+\mathbb{E}\left(X_{1}\right)
\end{aligned}
$$

Therefore using the fact that

$$
q e_{x}=q \cdot 1+q f_{x} \quad ; \quad\left\langle f_{x}, q e_{x}\right\rangle=-\left\langle q \cdot 1, f_{x}\right\rangle
$$

and equation (4.5), we obtain

$$
\begin{aligned}
& \left(A_{\nu}^{+}\left(q e_{x}\right)+A_{\nu}^{-}\left(q e_{x}\right)+\lambda(x)\right) \operatorname{Exp}\left(f_{x}\right)=A_{\nu}^{+}\left(q \cdot 1+q f_{x}\right) \operatorname{Exp}\left(f_{x}\right)+\left\langle q e_{x}, f_{x}\right\rangle \operatorname{Exp}\left(f_{x}\right) \\
& \quad-\left(\left\langle q e_{x}, f_{x}\right\rangle+\left\langle f_{x}, q e_{x}\right\rangle\right) \operatorname{Exp}\left(f_{x}\right)+\mathbb{E}\left(X_{1}\right) \operatorname{Exp}\left(f_{x}\right) \\
& \quad=A_{\nu}^{+}(q \cdot 1) \operatorname{Exp}\left(f_{x}\right)+A_{\nu}^{+}\left(q f_{x}\right) \operatorname{Exp}\left(f_{x}\right)-\left\langle f_{x}, q \cdot 1\right\rangle \operatorname{Exp}\left(f_{x}\right)+\mathbb{E}\left(X_{1}\right) \operatorname{Exp}\left(f_{x}\right) \\
& \quad=A_{\nu}^{+}(q) \operatorname{Exp}\left(f_{x}\right)+\Lambda_{\nu}(q) \operatorname{Exp}\left(f_{x}\right)+\left\langle q \cdot 1, f_{x}\right\rangle \operatorname{Exp}\left(f_{x}\right)+\mathbb{E}\left(X_{1}\right) \operatorname{Exp}\left(f_{x}\right) \\
& \quad=A_{\nu}^{+}(q \cdot 1) \operatorname{Exp}\left(f_{x}\right)+\Lambda_{\nu}(q) \operatorname{Exp}\left(f_{x}\right)+A_{\nu}^{-}(q \cdot 1) \operatorname{Exp}\left(f_{x}\right)+\mathbb{E}\left(X_{1}\right) \operatorname{Exp}\left(f_{x}\right)
\end{aligned}
$$

Finally, the previous equation and (4.10) yields

$$
\begin{aligned}
Q & =\left(A^{+}(-i b)+A^{-}(-i b)\right) \otimes 1+1 \otimes\left(A_{\nu}^{+}(q \cdot 1)+A_{\nu}^{-}(q \cdot 1)+\Lambda_{\nu}(q)+\mathbb{E}\left(X_{1}\right) \mathbf{1}\right) \\
& =Q_{G} \otimes 1+1 \otimes Q_{C P}
\end{aligned}
$$

\section{The weak quantum decomposition}

If the second order moment of $\nu$ does not exist the technique used in the proof of Theorem (4.1) cannot be applied.

In the present section we assume that only the first order moment of $\nu$ is finite, meaning by this that the vector $q \cdot 1 \in L^{1}(\nu)$ but $q \cdot 1 \notin L^{2}(\nu)$. This implies that for any $f \in L^{\infty}(\nu), q \cdot f \in L^{1}(\nu)$.

Under this assumption we prove that the quantum decomposition of the generalized field can be given a meaning in a weak sense (see Definition (5.3) below). 
Since the problem of infinite moments is only related to the Lévy measure, in this and the following section we will neglect the Gaussian part (i.e. we focus on the case $b=0)$ and for simplicity of notation we denote again $\Psi$ the characteristic exponent associated to the compound Poisson process.

If $\mu$ has only the first order moment, then $q \cdot 1 \notin L^{2}(\nu)$ and the first problem is to define objects like:

$$
A_{\nu}^{+}(q \cdot 1) \quad ; \quad A_{\nu}^{-}(q \cdot 1)
$$

To this goal recall that, denoting $U n\left(\mathcal{K}_{0}\right)$ the unitary group on $\mathcal{K}_{0}$, for any

$$
(u, T, v, z) \in \mathcal{K}_{0} \times U n\left(\mathcal{K}_{0}\right) \times K_{0} \times \mathbb{C}
$$

the operator

$$
\Gamma(u, T, v, z)=e^{A^{+}(u)} \Gamma(T) e^{A^{-}(v)} e^{z}
$$

is well-defined on the domain of the exponential vectors and maps the scalar multiples of these vectors into themselves because

$$
\Gamma(u, T, v, z) \operatorname{Exp}(f)=e^{z+\langle v, f\rangle} \operatorname{Exp}(T f+u) \quad ; \quad f \in \mathcal{K}_{0}
$$

Moreover the set (5.1) is a group for the composition law

$$
\left(u_{1}, T_{1}, v_{1}, z_{1}\right) \circ\left(u_{2}, T_{2}, v_{2}, z_{2}\right):=\left(u_{1}+T u_{2}, T_{1} T_{2}, v_{2}+T_{2}^{*} v_{1}, z_{1}+z_{2}+\left\langle v_{1}, u_{2}\right\rangle\right)
$$

(a generalization of the Heisenberg group) and the map

$$
(u, T, v, z) \in \mathcal{K}_{0} \times U n\left(\mathcal{K}_{0}\right) \times K_{0} \times \mathbb{C} \rightarrow \Gamma(u, T, v, z)
$$

is a group representation, i.e. $\forall u_{1}, u_{2}, v_{1}, v_{2} \in L^{2}(\nu), z_{1}, z_{2} \in \mathbb{C}$ and for any two unitary operators $T_{1}, T_{2}$ on $\mathcal{K}_{0}$ one has $\left(\mathrm{see}^{12}\right)$ :

$$
\Gamma\left(u_{1}, T_{1}, v_{1}, z_{1}\right) \Gamma\left(u_{2}, T_{2}, v_{2}, z_{2}\right)=\Gamma\left(u_{1}+T u_{2}, T_{1} T_{2}, v_{2}+T_{2}^{*} v_{1}, z_{1}+z_{2}+\left\langle v_{1}, u_{2}\right\rangle\right)
$$

Theorem 5.1. The operator valued function

$$
t \longmapsto W(t):=\Gamma\left(f_{t}, e^{i t q}, f_{-t}, \Psi(t)\right)=e^{A^{+}\left(f_{t}\right)} \Gamma\left(e^{i t q}\right) e^{A^{-}\left(f_{-t}\right)} e^{\Psi(t)}
$$

is a strongly continuous one-parameter unitary group with generator $Q_{C P}$.

\section{Proof.}

Step 1. It is known (and easily checked) that $\left(f_{t}\right)$ is a 1-cocycle for the group $\left(e^{i s q}\right)_{s \in \mathbb{R}}$, i.e.

$$
f_{t+s}=e^{i s q} f_{t}+f_{s} \quad, \quad f_{-t}=-e^{-i t q} f_{t}
$$

and that the 2-coboundary associated to $\Psi$ has the form

$$
\Psi(t+s)-\Psi(s)-\Psi(t)=\Psi(t-(-s))-\overline{\Psi(-s)}-\Psi(t)=q(-s, t)=\left\langle f_{-s}, f_{t}\right\rangle
$$

Therefore, for $f, g \in \mathcal{K}_{0}$, one has:

$$
\langle W(t) \operatorname{Exp}(f), W(t) \operatorname{Exp}(g)\rangle=
$$




$$
\begin{aligned}
& =\left\langle e^{\Psi(t)+\left\langle f_{-t}, f\right\rangle} \operatorname{Exp}\left(e^{i t q} f+f_{t}\right), e^{\Psi(t)+\left\langle f_{-t}, g\right\rangle} \operatorname{Exp}\left(e^{i t q} g+f_{t}\right)\right\rangle \\
& =\exp \left\{\overline{\Psi(t)}+\Psi(t)+\overline{\left\langle f_{-t}, f\right\rangle}+\left\langle f_{-t}, g\right\rangle\right\}\left\langle\operatorname{Exp}\left(e^{i t q} f+f_{t}\right), \operatorname{Exp}\left(e^{i t q} g+f_{t}\right)\right\rangle \\
& =\exp \left\{\overline{\Psi(t)}+\Psi(t)+\overline{\left\langle f_{-t}, f\right\rangle}+\left\langle f_{-t}, g\right\rangle+\left\langle e^{i t q} f+f_{t}, e^{i t q} g+f_{t}\right\rangle\right\} \\
& =\exp \left\{\left\langle f_{t}, f_{t}\right\rangle+\overline{\Psi(t)}+\Psi(t)+\overline{\left\langle-e^{-i t q} f_{t}, f\right\rangle}+\left\langle-e^{-i t q} f_{t}, g\right\rangle\right. \\
& \left.+\left\langle f, e^{-i t q} f_{t}\right\rangle+\left\langle f_{t}, e^{i t q} g\right\rangle+\langle f, g\rangle\right\} \\
& =\exp \{q(t, t)+\overline{\Psi(t)}+\Psi(t)+\langle f, g\rangle\} \\
& =\exp \{\langle f, g\rangle\} \\
& =\langle\operatorname{Exp}(f), \operatorname{Exp}(g)\rangle
\end{aligned}
$$

Thus $W(t)$ is unitary $\forall t \in \mathbb{R}$.

Step 2. Now we prove the group property and the strong continuity.

It is easily seen that

$$
W(0)=\Gamma\left(f_{0}, \mathbf{1}, f_{0}, \Psi(0)\right)=\mathbf{1}
$$

and we have

$$
\begin{aligned}
W(t) W(s) & =\Gamma\left(f_{t}, e^{i t q}, f_{-t}, \Psi(t)\right) \Gamma\left(f_{s}, e^{i s q}, f_{-s}, \Psi(s)\right) \\
& =\Gamma\left(f_{t}+e^{i t q} f_{s}, e^{i t q} e^{i s q}, f_{-s}+\left(e^{i s q}\right)^{*} f_{-t}, \Psi(t)+\Psi(s)+\left\langle f_{-t}, f_{s}\right\rangle\right)
\end{aligned}
$$

From the identities

$$
\begin{aligned}
f_{t}+e^{i t q} f_{s}=f_{t+s} ; & f_{-s}+\left(e^{i s q}\right)^{*} f_{-t}=f_{-s}+e^{-i s q} f_{-t}=f_{-s-t} \\
\Psi(t)+\Psi(s)+\left\langle f_{-t}, f_{s}\right\rangle= & \Psi(t)+\Psi(s)+q(-t, s) \\
= & \Psi(t)+\Psi(s)+\Psi(s-(-t))-\overline{\Psi(-t)}-\Psi(s) \\
= & \Psi(t+s)
\end{aligned}
$$

we deduce that

$$
W(t) W(s)=\Gamma\left(f_{t+s}, e^{i(t+s) q}, f_{-(t+s)}, \Psi(t+s)\right)=W(t+s)
$$

For the strong continuity, it is sufficient to prove that

$$
\lim _{t \rightarrow 0}\|W(t) \operatorname{Exp}(f)-\operatorname{Exp}(f)\|=0 \quad \forall f \in L^{2}(\nu)
$$


We have

$$
\begin{aligned}
\|W(t) \operatorname{Exp}(f)-\operatorname{Exp}(f)\|^{2}= & \|W(t) \operatorname{Exp}(f)\|^{2}+\|\operatorname{Exp}(f)\|^{2} \\
- & 2 \Re\langle\operatorname{Exp}(f), W(t) \operatorname{Exp}(f)\rangle \\
= & 2\|\operatorname{Exp}(f)\|^{2}-2 \Re\langle\operatorname{Exp}(f), W(t) \operatorname{Exp}(f)\rangle \\
= & 2 e^{\|f\|^{2}}-2 \Re\left(\left\langle\operatorname{Exp}(f), \exp \left(\Psi(t)+\left\langle f_{-t}, f\right\rangle\right)\right.\right. \\
& \left.\left.\left.\operatorname{Exp}^{i t q} f+f_{t}\right)\right\rangle\right) \\
= & 2 e^{\|f\|^{2}}-2 \Re\left(\operatorname { e x p } \left(\Psi(t)+\left\langle f_{-t}, f\right\rangle\right.\right. \\
& \left.\left.+\left\langle f, e^{i t q} f\right\rangle+\left\langle f, f_{t}\right\rangle\right)\right) .
\end{aligned}
$$

By dominated convergence $\forall f \in \mathcal{K}_{0}$ one has

$$
\left\langle f, e^{i t q} f\right\rangle=\int_{\mathbb{R} \backslash\{0\}}|f(s)|^{2} e^{i t s} \nu(d s) \longrightarrow\|f\|^{2} \quad \text { as } \quad(t \rightarrow 0)
$$

On the other hand for $|t|<1$, one has also

$$
\left|f(s)\left(e^{i s t}-1\right)\right| \leq \varphi(s)=\left\{\begin{array}{l}
|s f(s)| \text { if }|s| \leq 1 \\
2|f(s)| \text { if }|s|>1
\end{array}\right.
$$

and $\varphi \in L^{1}(\nu)$ because

$$
\begin{aligned}
\int_{\mathbb{R} \backslash\{0\}} \varphi(s) \nu(d s) & =\int_{|s| \leq 1}|s f(s)| \nu(d s)+\int_{|s|>1} 2|f(s)| \nu(d s) \\
& \leq\left(\int_{|s| \leq 1} s^{2} \nu(d s)\right)^{\frac{1}{2}}\left(\int_{|s| \leq 1}|f(s)|^{2} \nu(d s)\right)^{\frac{1}{2}} \\
& +2\left(\int_{|s|>1} \nu(d s)\right)^{\frac{1}{2}}\left(\int_{|s|>1}|f(s)|^{2} \nu(d s)\right)^{\frac{1}{2}}<\infty
\end{aligned}
$$

Again by dominated convergence we conclude that

$$
\lim _{t \rightarrow 0}\left\langle f, f_{t}\right\rangle=\int_{\mathbb{R} \backslash\{0\}} \lim _{t \rightarrow 0} \overline{f(s)}\left(e^{i s t}-1\right) \nu(d s)=0 .
$$

Taking the limit $t \rightarrow 0$ in Eq. (5.5) and using (5.6) and (5.7) one obtains

$$
\lim _{t \rightarrow 0}\|W(t) \operatorname{Exp}(f)-\operatorname{Exp}(f)\|^{2}=\lim _{t \rightarrow 0} 2\left(e^{\|f\|^{2}}-\Re\left(e^{\|f\|^{2}}\right)\right)=0 .
$$


Finally from (4.6) one sees that, for all $t \in \mathbb{R}$

$$
\begin{aligned}
W(t) \operatorname{Exp}\left(f_{x}\right) & =\exp \left(\Psi(t)+\left\langle f_{-t}, f_{x}\right\rangle\right) \operatorname{Exp}\left(e^{i t q} f_{x}+f_{t}\right) \\
& =\exp (\Psi(t)+q(-t, x)) \operatorname{Exp}\left(f_{x+t}\right) \\
& =\exp (\Psi(x+t)-\Psi(x)) \operatorname{Exp}\left(f_{x+t}\right) \\
& =e^{i t Q_{C P}} \operatorname{Exp}\left(f_{x}\right)
\end{aligned}
$$

\subsection{Distribution valued operators}

In this section we extend the definition of creation, annihilation and preservation operators to include the case when the images of some vectors in the Fock space are not vectors of the same space but elements in the algebraic dual of a dense subspace of it. In this sense we speak of distribution valued operators. Using a variant of the Hida-Kubo-Takenaka construction it is possible to introduce topologies such that the action of these operators become continuous, but this construction, not needed in the present paper, will be discussed elsewhere.

We emphasize, for future use, that all the arguments and constructions in the present sub-section do not require that $q \cdot 1 \in L^{1}(\nu)$.

Definition 5.1. A distribution valued operator $T$ on a Hilbert space $\mathcal{H}$ with dense domain $\mathcal{D}$ is a linear map from $\mathcal{D} \subseteq \mathcal{H}$ to its algebraic dual $\mathcal{D}^{\prime}$.

Remark 5.1. The natural embedding

$$
\xi \in \mathcal{H} \mapsto\langle\xi, \cdot\rangle \in \mathcal{H}^{\prime} \subset \mathcal{D}^{\prime}
$$

allows to adopt the language of standard triplets

$$
\mathcal{D} \subset \mathcal{H} \subset \mathcal{D}^{\prime}
$$

and to interpret the elements of $\mathcal{D}^{\prime}$ as vector valued distributions on $\mathcal{D}$.

Let $\mathcal{C}_{\nu}$ be a total set in $\mathcal{K}_{0}$ with the following properties:

(C.i) $\mathcal{C}_{\nu}$ is invariant under complex conjugate,

(C.ii) for all $g \in \mathcal{C}_{\nu}$, the distributions

$$
q \cdot 1: f \longmapsto\langle q \cdot 1, f\rangle \quad \text { and } \quad q \cdot g: f \longmapsto\langle q \cdot g, f\rangle=\langle q \cdot 1, f \bar{g}\rangle
$$

are well-defined on $\operatorname{Lin}-\operatorname{span}\left(\mathcal{C}_{\nu}\right)$. 
Definition 5.2. For $f \in \mathcal{C}_{\nu}$, define the operators $A_{\nu}^{-}(q \cdot 1)$ and $A_{\nu}^{-}(q f)$ on the domain $\operatorname{Exp}\left(\mathcal{C}_{\nu}\right) \subseteq \Gamma\left(\mathcal{K}_{0}\right)$ (the linear subspace of $\Gamma\left(\mathcal{K}_{0}\right)$ generated by $\{\operatorname{Exp}(g)$ : $\left.\left.g \in \mathcal{C}_{\nu}\right\}\right)$ by linear extension of:

$$
A_{\nu}^{-}(q \cdot 1) \operatorname{Exp}(g):=\langle q \cdot 1, g\rangle \operatorname{Exp}(g) \quad ; \quad g \in \mathcal{C}_{\nu}
$$

and

$$
A_{\nu}^{-}(q f) \operatorname{Exp}(g):=\langle q \cdot 1, \bar{f} g\rangle \operatorname{Exp}(g) \quad ; \quad g \in \mathcal{C}_{\nu}
$$

respectively.

Define the distribution valued operators $A_{\nu}^{+}(q \cdot 1)$ and $\Lambda_{\nu}(q)$ on the domain $\operatorname{Exp}\left(\mathcal{C}_{\nu}\right) \subset \Gamma\left(\mathcal{K}_{0}\right)$ by the prescription that, for each $f, g \in \mathcal{C}_{\nu}$ one has:

$$
\begin{gathered}
\left\langle A_{\nu}^{+}(q \cdot 1) \operatorname{Exp}(f), \operatorname{Exp}(g)\right\rangle:=\left\langle\operatorname{Exp}(f), A_{\nu}^{-}(q \cdot 1) \operatorname{Exp}(g)\right\rangle \\
\left\langle\Lambda_{\nu}(q) \operatorname{Exp}(f), \operatorname{Exp}(g)\right\rangle:=\left\langle\operatorname{Exp}(f), A_{\nu}^{-}(q f) \operatorname{Exp}(g)\right\rangle
\end{gathered}
$$

Remark 5.2. One easily proves that the definition (5.11) of $\Lambda_{\nu}(q)$ is compatible with the usual one, as the differential second quantization of $q$, in the sense that the two definitions coincide on the set of exponential vectors with test functions in the domain of $q$.

\subsection{Weak domains}

Definition 5.3. Let $U(t)=e^{i t A}$ be a strongly continuous one-parameter unitary group on a Hilbert space $\mathcal{H}$ with generator $A$. Define the weak domain wk-dom $(A)$ of $A$ as the maximal subspace $\mathcal{D}$ of $\mathcal{H}$ such that $\forall \varphi, \phi \in \mathcal{D}$ the limit

$$
\lim _{t \rightarrow 0}\left\langle\frac{U(t)-1}{t} \varphi, \phi\right\rangle
$$

exists.

Remark 5.3. Clearly the weak domain of $A$ contains the domain of $A$. In particular wk- $\operatorname{dom}(A)$ is a dense subspace of $\mathcal{H}$ and $A$ can be defined as a distribution valued operator on its weak domain by the prescription:

$$
A \psi:=i \lim _{t \rightarrow 0}\left\langle\frac{U(t)-1}{t} \psi, \cdot\right\rangle
$$

Lemma 5.1. Let $\mathcal{C}_{\nu}:=\mathcal{E}=\left\{f_{x}, x \in \mathbb{R}\right\} \subset \mathcal{K}_{0}$. Then $\mathcal{C}_{\nu}$ is a total set in $\mathcal{K}_{0}$ satisfying conditions $(\mathbf{C . i})$ and (C.ii). Moreover for all $f \in \mathcal{C}_{\nu}$, the function

$$
F: t \longmapsto\left\langle f_{t}, f\right\rangle
$$

is derivable at $t=0$ and

$$
F^{\prime}(0)=-i\langle q \cdot 1, f\rangle:=-i \int_{\mathbb{R} \backslash\{0\}} t f(t) \nu(d t) .
$$

Proof. The totality is satisfied by definition of $\mathcal{K}_{0}$.

From relation $\bar{f}_{x}=f_{-x}, x \in \mathbb{R}$, we deduce that $\mathcal{C}_{\nu}$ is invariant under complex 
conjugate. Then condition (C.i) holds.

While the first order moment of $\nu$ exists, then $\left(t \longmapsto t f_{x}(t)\right) \in L^{1}(\nu)$. In fact $\left|t f_{x}(t)\right| \leq 2|t|$. This gives that

$$
\left\langle q \cdot 1, f_{x}\right\rangle:=\int_{\mathbb{R} \backslash\{0\}} t f_{x}(t) \nu(d t)
$$

exists for all $x \in \mathbb{R}$. Then by a linear extension, the distribution

$$
\mathcal{C}_{\nu} \ni f \longmapsto\langle q \cdot 1, f\rangle:=\int_{\mathbb{R} \backslash\{0\}} t f(t) \nu(d t)
$$

is well-defined.

From relation $f_{x} f_{y}=f_{x+y}-f_{x}-f_{y}, x, y \in \mathbb{R}$, we deduce that $\mathcal{C}_{\nu}$ is invariant under multiplication and it is the same for $\operatorname{Lin}-\operatorname{span}\left(\mathcal{C}_{\nu}\right)$. Then for all $g \in \mathcal{C}_{\nu}$, the distribution

$$
\operatorname{Lin}-\operatorname{span}\left(\mathcal{C}_{\nu}\right) \ni f \longmapsto\langle q \cdot 1, f \bar{g}\rangle:=\int_{\mathbb{R} \backslash\{0\}} t f(t) \overline{g(t)} \nu(d t)
$$

is well-defined also which proves that Condition (C.ii) is satisfied.

Let $x \in \mathbb{R}$, defining the function

$$
t \longmapsto F(t):=\left\langle f_{t}, f_{x}\right\rangle=\int_{\mathbb{R} \backslash\{0\}} \overline{f_{t}(s)} f_{x}(s) \nu(d t) .
$$

First $F$ is well-defined because $f_{t}, f_{x} \in L^{2}(\nu)$. On the other hand

$$
\left|\frac{\partial}{\partial t}\left(\overline{f_{t}(s)} f_{x}(s)\right)\right|=\left|-i s e^{-i s t} f_{x}(s)\right|=\left|s f_{x}(s)\right| \in L^{1}(\nu) .
$$

Then $F$ is derivable at any $t \in \mathbb{R}$ and

$$
F^{\prime}(t)=-i \int_{\mathbb{R} \backslash\{0\}} s e^{-i t s} f_{x}(s) \nu(d t),
$$

in particular $F^{\prime}(0)=-i\left\langle q \cdot 1, f_{x}\right\rangle$.

In the remain of this section, we take $\mathcal{C}_{\nu}:=\left\{f_{x}, x \in \mathbb{R}\right\}$.

Theorem 5.2. The exponential vectors $\operatorname{Exp}\left(\mathcal{C}_{\nu}\right)$ are in the weak domain $w k-\operatorname{dom}\left(Q_{C P}\right)$ of $Q_{C P}$. Moreover, on the domain $\operatorname{Exp}\left(\mathcal{C}_{\nu}\right)$, the operator $Q_{C P}$ coincides with the distribution valued operator

$$
A_{\nu}^{+}(q \cdot 1)+A_{\nu}^{-}(q \cdot 1)+\Lambda_{\nu}(q)+\mathbb{E}\left(X_{1}\right) \mathbf{1}
$$

Proof. From Theorem (5.1) one knows that $Q_{C P}$ is the generator of $W(t)$ and that:

$$
\begin{aligned}
\left\langle W(t) \operatorname{Exp}\left(f_{x}\right), \operatorname{Exp}\left(f_{y}\right)\right\rangle & =\left\langle e^{\Psi(t)+\left\langle f_{-t}, f_{x}\right\rangle} \operatorname{Exp}\left(f_{t}+e^{i t q} f_{x}\right), \operatorname{Exp}\left(f_{y}\right)\right\rangle \\
& =\exp \left(\Psi(-t)+\left\langle f_{x}, f_{-t}\right\rangle+\left\langle f_{t}, f_{y}\right\rangle+\left\langle e^{i t q} f_{x}, f_{y}\right\rangle\right) \\
& =e^{h(t)}
\end{aligned}
$$


where

$$
\begin{aligned}
h(t) & :=\Psi(-t)+\left\langle f_{x}, f_{-t}\right\rangle+\left\langle f_{t}, f_{y}\right\rangle+\left\langle e^{i t q} f_{x}, f_{y}\right\rangle \\
& =\Psi(-t)+\left\langle f_{t}, f_{-x}\right\rangle+\left\langle f_{t}, f_{y}\right\rangle+\left\langle f_{t}, f_{-x} f_{y}\right\rangle+\left\langle f_{x}, f_{y}\right\rangle \\
& =\Psi(-t)+\left\langle f_{t}, f_{-x}+f_{y}+f_{-x} f_{y}\right\rangle+\left\langle f_{x}, f_{y}\right\rangle \\
& =\Psi(-t)+\left\langle f_{t}, f_{y-x}\right\rangle+\left\langle f_{x}, f_{y}\right\rangle
\end{aligned}
$$

Lemma (5.1), proves that the function $h$ is derivable at $(t=0)$ with

$$
\begin{aligned}
h^{\prime}(0) & =-\psi^{\prime}(0)-i\left\langle q \cdot 1, f_{y-x}\right\rangle \\
& =-i\left(\mathbb{E}\left(X_{1}\right)+\left\langle q \cdot 1, f_{y-x}\right\rangle\right) \\
& =-i\left(\mathbb{E}\left(X_{1}\right)+\overline{\left\langle q \cdot 1, f_{x}\right\rangle}+\left\langle q \cdot 1, f_{y}\right\rangle+\left\langle q \cdot 1, \overline{f_{x}} f_{y}\right\rangle\right) .
\end{aligned}
$$

Then

$$
\begin{aligned}
\lim _{t \rightarrow 0}\left\langle\frac{W(t)-1}{t} \operatorname{Exp}\left(f_{x}\right), \operatorname{Exp}\left(f_{y}\right)\right\rangle & =\left.\frac{d}{d t}\right|_{t=0}\left\langle W(t) \operatorname{Exp}\left(f_{x}\right), \operatorname{Exp}\left(f_{y}\right)\right\rangle \\
& =h^{\prime}(0) e^{h(0)}
\end{aligned}
$$

Therefore for each $f_{x} \in \mathcal{C}_{\nu}, \operatorname{Exp}\left(f_{x}\right) \in$ wk- $\operatorname{dom}\left(Q_{C P}\right)$ i.e., $\operatorname{Exp}\left(\mathcal{C}_{\nu}\right) \subset$ wk- $\operatorname{dom}\left(Q_{C P}\right)$.

We know that $\operatorname{Exp}\left(\mathcal{C}_{\nu}\right)$ is in the domain of the operator valued distributions $A_{\nu}^{-}(q \cdot 1), A_{\nu}^{+}(q \cdot 1), \Lambda_{\nu}(q)$ and one has:

$$
\begin{aligned}
& \left\langle Q_{C P} \operatorname{Exp}\left(f_{x}\right), \operatorname{Exp}\left(f_{y}\right)\right\rangle \\
= & i \lim _{t \rightarrow 0}\left\langle\frac{W(t)-1}{t} \operatorname{Exp}\left(f_{x}\right), \operatorname{Exp}\left(f_{y}\right)\right\rangle \\
= & i h^{\prime}(0) e^{h(0)} \\
= & \left(\mathbb{E}\left(X_{1}\right)+\overline{\left\langle q \cdot 1, f_{x}\right\rangle}+\left\langle q \cdot 1, f_{y}\right\rangle+\left\langle q \cdot 1, \overline{f_{x}} f_{y}\right\rangle\right) e^{\left\langle f_{x}, f_{y}\right\rangle} \\
= & \left\langle\mathbb{E}\left(X_{1}\right) \operatorname{Exp}\left(f_{x}\right), \operatorname{Exp}\left(f_{y}\right)\right\rangle+\left\langle\left\langle q \cdot 1, f_{x}\right\rangle \operatorname{Exp}\left(f_{x}\right), \operatorname{Exp}\left(f_{y}\right)\right\rangle \\
+ & \left\langle\operatorname{Exp}\left(f_{x}\right),\left\langle q \cdot 1, f_{y}\right\rangle \operatorname{Exp}\left(f_{y}\right)\right\rangle+\left\langle\operatorname{Exp}\left(f_{x}\right),\left\langle q \cdot 1, \bar{f}_{x} f_{y}\right\rangle \operatorname{Exp}\left(f_{y}\right)\right\rangle \\
= & \left\langle\mathbb{E}\left(X_{1}\right) \operatorname{Exp}\left(f_{x}\right), \operatorname{Exp}\left(f_{y}\right)\right\rangle+\left\langle A_{\nu}^{-}(q \cdot 1) \operatorname{Exp}\left(f_{x}\right), \operatorname{Exp}\left(f_{y}\right)\right\rangle \\
+ & \left\langle\operatorname{Exp}\left(f_{x}\right), A_{\nu}^{-}(q \cdot 1) \operatorname{Exp}\left(f_{y}\right)\right\rangle+\left\langle\operatorname{Exp}\left(f_{x}\right), A_{\nu}^{-}\left(q f_{x}\right) \operatorname{Exp}\left(f_{y}\right)\right\rangle
\end{aligned}
$$




$$
\begin{aligned}
& =\left\langle\mathbb{E}\left(X_{1}\right) \operatorname{Exp}\left(f_{x}\right), \operatorname{Exp}\left(f_{y}\right)\right\rangle+\left\langle A_{\nu}^{-}(q \cdot 1) \operatorname{Exp}\left(f_{x}\right), \operatorname{Exp}\left(f_{y}\right)\right\rangle \\
& +\left\langle A_{\nu}^{+}(q \cdot 1) \operatorname{Exp}\left(f_{x}\right), \operatorname{Exp}\left(f_{y}\right)\right\rangle+\left\langle\Lambda_{\nu}(q) \operatorname{Exp}\left(f_{x}\right), \operatorname{Exp}\left(f_{y}\right)\right\rangle \\
& =\left\langle\left(\mathbb{E}\left(X_{1}\right)+A_{\nu}^{-}(q \cdot 1)+A_{\nu}^{+}(q \cdot 1)+\Lambda_{\nu}(q)\right) \operatorname{Exp}\left(f_{x}\right), \operatorname{Exp}\left(f_{y}\right)\right\rangle
\end{aligned}
$$

\section{The renormalized quantum decomposition}

In this section we assume the non existence of the first order moment of $\nu$, i.e. that $q \cdot 1 \notin L^{1}(\nu)$.

Comparing the expressions (5.12) and (4.9) one sees that, even if they they should be understood in different ways, they look formally the same and that the existence of a finite first order moment is a necessary condition for both expressions to make sense. Therefore, for random variables not satisfying this condition, one must look for a notion of quantum decomposition different from the one given by expressions of the form (4.9).

On the other hand the already emphasized fact, that all the results of section (5.1) do not require the existence of the first order moment, suggests that all the problems with the extension of expression (4.9) to random variables without moments is concentrated on the scalar term in the sense that, after subtracting this term, which is infinite in the case of random variables without moments, one obtains the meaningful distribution valued operator

$$
A_{\nu}^{+}(q \cdot 1)+A_{\nu}^{-}(q \cdot 1)+\Lambda_{\nu}(q)
$$

In physics the procedure of subtracting infinite constants to some expressions, in order to transform them into meaningful and physically measurable ones, is well known and called additive renormalization.

In the present case a mere additive renormalization would not be sufficient because it would leave open the question of the connection between the resulting expression (6.1) after additive renormalization and the original random variable without moments. In other words, we want the renormalized quantum decomposition (6.1) to be canonically associated to the the random variable $Q_{C P}$ or equivalently to the 1-parameter group exp $i t Q_{C P}$ generated by it.

In the following we prove that such a canonical connection can be established using a multiplicative renormalization procedure. In mathematical terms this means the transition from a representation of the additive group $\mathbb{R}$ to a projective representation of the same group.

The idea of the construction of this projective representation is naturally suggested by the proof of Theorem (5.2). In fact from it one can see that the emergence of the first moment in the quantum decomposition is due to the derivative of the 
scalar term in the normally ordered form (5.4) of the one-parameter unitary group $\exp i t Q_{C P}$, i.e. $\exp \Psi(t)$. Therefore the emergence of the "infinite constant" $\mathbb{E}\left(X_{1}\right)$ in the formal expression (5.12) is a manifestation of the fact that, if the first moment of the random variable $X_{1}$ is infinite, then the function $\Psi$ is not differentiable.

In order to remove this constant from $\exp i t Q_{C P}$ notice that, if $\left(W_{t}\right)_{t \in \mathbb{R}}$ is a unitary representation of $\mathbb{R}$ and $t \in \mathbb{R} \longmapsto \mu_{t} \in \mathbb{C}$ is any measurable function, then the 1-parameter family

$$
V_{t}:=e^{-\mu_{t}} W_{t}
$$

is a projective, in general non unitary, representation of $\mathbb{R}$ with multiplier (which is in fact a $2-$-co-boundary):

$$
\hat{\sigma}(s, t):=e^{\mu_{t+s}-\mu_{s}-\mu_{t}}
$$

In other terms:

$$
V_{s} V_{t}=\hat{\sigma}(s, t) V_{s+t} \quad ; s, t, \in \mathbb{R}
$$

Now we apply this remark to the case when $\mu_{t}=\Psi(t)$ and $W_{t}$ is given by (5.5).

Lemma 6.1. Let $p$ be the orthogonal projection on $\mathcal{K}_{0}$ and $C_{c, 0}^{\infty}$ be the dense subspace of $L^{2}(\nu)$ of the infinitely differentiable functions with compact support non containing zero. Let

$$
\mathcal{C}_{\nu}:=p\left(C_{c, 0}^{\infty}\right)=\left\{p(\varphi), \quad \varphi \in C_{c, 0}^{\infty}\right\}
$$

be the orthogonal projection of $C_{c, 0}^{\infty}$ on $\mathcal{K}_{0}$. Then $\mathcal{C}_{\nu}$ is a dense sub-space of $\mathcal{K}_{0}$ satisfying properties $(\mathbf{C . i})$ and $(\mathbf{C} . \mathbf{i i})$.

\section{Proof.}

i Density:

Let $f \in \mathcal{K}_{0} \subset L^{2}(\nu)$, by the density of $C_{c, 0}^{\infty}$ in $L^{2}(\nu)$, there exists $\varphi_{n} \in C_{c, 0}^{\infty}$ converging to $f$ as $n \longrightarrow+\infty$.

But by definition of $p, \varphi_{n}-p\left(\varphi_{n}\right) \perp p\left(\varphi_{n}\right)-f \in \mathcal{K}_{0}$, then

$$
\begin{aligned}
\left\|\varphi_{n}-f\right\|^{2} & =\left\|\left(\varphi_{n}-p\left(\varphi_{n}\right)\right)+\left(p\left(\varphi_{n}\right)-f\right)\right\|^{2} \\
& =\left\|\varphi_{n}-p\left(\varphi_{n}\right)\right\|^{2}+\left\|p\left(\varphi_{n}\right)-f\right\|^{2}
\end{aligned}
$$

and we get

$$
\left\|p\left(\varphi_{n}\right)-f\right\| \leq\left\|\varphi_{n}-f\right\| \longrightarrow 0 \quad \text { as } \quad(n \longrightarrow+\infty) .
$$

Hence $f$ is a limit of a sequence of $\mathcal{C}_{\nu}$ which proves the density.

ii Condition (C.i):

Let $f=p(\varphi) \in \mathcal{C}_{\nu}$ where $\varphi \in C_{c, 0}^{\infty}$. While $C_{c, 0}^{\infty}$ is invariant under complex conjugate, then to prove this property for $\mathcal{C}_{\nu}$, it is sufficient to prove that 
$\bar{f}=p(\bar{\varphi})$.

We have $f-\varphi \in \mathcal{K}_{0}^{\perp}=\left\{f_{x}, x \in \mathbb{R}\right\}^{\perp}$, then

$$
\left\langle\bar{f}-\bar{\varphi}, f_{x}\right\rangle=\left\langle f_{-x}, f-\varphi\right\rangle=0 \quad \forall x \in \mathbb{R} .
$$

This gives $\bar{f}-\bar{\varphi} \in \mathcal{K}_{0}^{\perp}$. But $\bar{f} \in \mathcal{K}_{0}$. Then $\bar{f}=p(\bar{\varphi}) \in \mathcal{C}_{\nu}$.

iii Condition (C.ii):

For $f \in \mathcal{C}_{\nu}$, let us consider the function $F_{f}(x)=\left\langle f_{x}, f\right\rangle$. Let $\varphi \in C_{c, 0}^{\infty}$ such that $f=p(\varphi)$. Then $f-\varphi \in \mathcal{K}_{0}^{\perp}$ which gives $F_{f}(x)=\left\langle f_{x}, \varphi\right\rangle$. It is easily checked that $F_{f}$ is derivable at $x=0$ and

$$
F_{f}^{\prime}(0)=-i \int_{\mathbb{R} \backslash\{0\}} t \varphi(t) \nu(d t)=-i\langle q \cdot 1, \varphi\rangle .
$$

Clearly that $\langle q \cdot 1, \varphi\rangle$ dose not depend on the choice of $\varphi$ but only on $f$. Then the distribution

$$
f \longmapsto\langle q \cdot 1, f\rangle:=\langle q \cdot 1, \varphi\rangle=i F_{f}^{\prime}(0) .
$$

is well-defined. Hence the first part of Condition (C.ii) is proved.

Let us proving the second part.

First notice that for all $g \in \mathcal{K}_{0}, f_{x} g \in \mathcal{K}_{0}$. In fact $g$ is a limit in $L^{2}(\nu)$ of a sequence $\left(g_{n}\right)_{n} \subset \operatorname{Lin}-\operatorname{span}(\mathcal{E})$, then $f_{x} g_{n} \in \operatorname{Lin}-\operatorname{span}(\mathcal{E})$. Moreover

$$
\begin{aligned}
\left\|f_{x} g_{n}-f_{x} g\right\|^{2} & =\int_{\mathbb{R} \backslash\{0\}}\left|f_{x}(t)\left(g_{n}(t)-g(t)\right)\right|^{2} \nu(d t) \\
& \leq 4\left\|g_{n}-g\right\|^{2} \longrightarrow 0 \quad(\text { as } \quad n \longrightarrow+\infty) .
\end{aligned}
$$

Hence $f_{x} g$ is a limit of a some sequence of $\operatorname{Lin}-\operatorname{span}(\mathcal{E})$, then it belongs to $\mathcal{K}_{0}$.

Let $f=p(\varphi) \in \mathcal{C}_{\nu}$, where $\varphi \in C_{c, 0}^{\infty}$. Then by definition of $p,(f-\varphi) \perp f_{x} g$ which gives

$$
\left\langle f_{x}, f \bar{g}-\varphi \bar{g}\right\rangle=\left\langle f_{x} g, f-\varphi\right\rangle=0 .
$$

Let consider the function

$$
G_{g, f}(x):=\left\langle f_{x}, f \bar{g}\right\rangle=\left\langle f_{x}, \varphi \bar{g}\right\rangle=\int_{\mathbb{R} \backslash\{0\}} f_{-x}(t) \overline{g(t)} \varphi(t) \nu(d t)
$$

Clearly that $G_{g, f}$ is derivable at $x=0$ and

$$
G_{g, f}^{\prime}(0)=-i \int_{\mathbb{R} \backslash\{0\}} t \overline{g(t)} \varphi(t) \nu(d t)
$$

Defining

$$
\langle q \cdot g, f\rangle:=\int_{\mathbb{R} \backslash\{0\}} t \overline{g(t)} \varphi(t) \nu(d t)=i G_{g, f}^{\prime}(0) .
$$

Clearly that $\langle q \cdot g, f\rangle$ does not depend of the choice of $\varphi$. Then the distribution

$$
f \longmapsto\langle q \cdot g, f\rangle
$$


is well defined on $\mathcal{C}_{\nu}$.

Theorem 6.1. Let $W(t)$ be the one-parameter unitary group defined by (5.5) and define:

$$
V(t):=e^{-\Psi(t)} W(t)=e^{A^{+}\left(f_{t}\right)} e^{\Lambda(i t q)} e^{A^{-}\left(f_{-t}\right)}
$$

Then $\{V(t) ; t \in \mathbb{R}\}$ is a strongly continuous projective representation of $\mathbb{R}$ with multiplier

$$
\hat{\sigma}(s, t):=e^{\Psi(s+t)-\Psi(s)-\Psi(t)}
$$

Its generator $Q_{\Psi}$ contains $\operatorname{Exp}\left(\mathcal{C}_{\nu}\right)$ in its weak domain and, on $\operatorname{Exp}\left(\mathcal{C}_{\nu}\right)$, coincides with

$$
A_{\nu}^{+}(q \cdot 1)+A_{\nu}^{-}(q \cdot 1)+\Lambda_{\nu}(q)
$$

Proof. Since $W(t)$ is a strongly continuous one-parameter unitary group and $\Psi$ is continuous, the strong continuity of $V(t)$ is clear because, for any $\varphi \in \mathcal{K}_{0}$ and $t_{0} \in \mathbb{R}$, denoting $\varphi_{0}:=V\left(t_{0}\right) \varphi$ one has as $s \rightarrow 0$ :

$$
\begin{aligned}
\left\|V\left(t_{0}+s\right) \varphi-V\left(t_{0}\right) \varphi\right\| & =\left\|e^{-\Psi\left(t_{0}+s\right)} W\left(t_{0}+s\right) \varphi-\varphi_{0}\right\| \\
& =\left\|e^{-\Psi\left(t_{0}+s\right)+\Psi\left(t_{0}\right)} W(s) \varphi_{0}-\varphi_{0}\right\| \\
& \leq\left\|W(s)\left(e^{-\Psi\left(s+t_{0}\right)+\Psi\left(t_{0}\right)} \varphi_{0}-\varphi_{0}\right)\right\|+\left\|W(s) \varphi_{0}-\varphi_{0}\right\| \\
& =\left\|e^{\Psi\left(t_{0}\right)-\Psi\left(t_{0}+s\right)} \varphi_{0}-\varphi_{0}\right\|+\left\|W(s) \varphi_{0}-\varphi_{0}\right\| \longrightarrow 0
\end{aligned}
$$

Let $f, g \in \mathcal{C}_{\nu}$, we have

$$
\begin{aligned}
& \left\langle\frac{V(t)-1}{t} \operatorname{Exp}(f), \operatorname{Exp}(g)\right\rangle=\frac{1}{t}\left(\langle V(t) \operatorname{Exp}(f), \operatorname{Exp}(g)\rangle-e^{\langle f, g\rangle}\right) \\
& =\frac{1}{t}\left(\left\langle e^{\overline{\left\langle f_{-t}, f\right\rangle}} \operatorname{Exp}\left(e^{i t q} f+f_{t}\right), \operatorname{Exp}(g)\right\rangle-e^{\langle f, g\rangle}\right)
\end{aligned}
$$

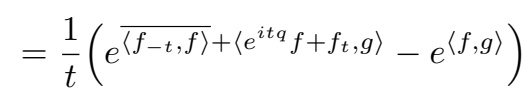

$$
\begin{aligned}
& =\frac{1}{t}\left(e^{h(t)}-e^{h(0)}\right)
\end{aligned}
$$

where in the notation of proof of Lemma (6.1)

$$
\begin{aligned}
h(t) & =\overline{\left\langle f_{-t}, f\right\rangle}+\left\langle e^{i t q} f+f_{t}, g\right\rangle \\
& =\overline{\left\langle f_{-t}, f\right\rangle}+\left\langle f_{t} f, g\right\rangle+\langle f, g\rangle+\left\langle f_{t}, g\right\rangle \\
& =\overline{F_{f}(-t)}+G_{f, g}(t)+F_{g}(t)+\langle f, g\rangle
\end{aligned}
$$


But it is clear from above calculations that $h$ is derivable at $(t=0)$ and

$$
\begin{aligned}
h^{\prime}(0) & =-\overline{F_{f}^{\prime}(0)}+F_{g}^{\prime}(0)+G_{f, g}^{\prime}(0) \\
& =-i(\overline{\langle q \cdot 1, f\rangle}+\langle q \cdot 1, g\rangle+\langle q \cdot 1, \bar{f} g\rangle)
\end{aligned}
$$

then the $\lim _{t \rightarrow 0}\left\langle\frac{V(t)-1}{t} \operatorname{Exp}(f), \operatorname{Exp}(g)\right\rangle$ exist. Hence $\operatorname{Exp}(f) \in w k-\operatorname{Dom}\left(Q_{\Psi}\right)$ and

$$
\begin{aligned}
\left\langle Q_{\Psi} \operatorname{Exp}\right. & (f), \operatorname{Exp}(g)\rangle \\
= & i\left\langle\lim _{t \rightarrow 0} \frac{V(t)-1}{t} \operatorname{Exp}(f), \operatorname{Exp}(g)\right\rangle \\
& =i h^{\prime}(0) e^{h(0)} \\
& =(\overline{\langle q \cdot 1, f\rangle}+\langle q \cdot 1, g\rangle+\langle q \cdot 1, \bar{f} g\rangle) e^{\langle f, g\rangle} \\
& =\langle\langle q \cdot 1, f\rangle \operatorname{Exp}(f), \operatorname{Exp}(g)\rangle+\langle\operatorname{Exp}(f),\langle q \cdot 1, g\rangle \operatorname{Exp}(g)\rangle \\
& +\langle\operatorname{Exp}(f),\langle q \cdot 1, \bar{f} g\rangle \operatorname{Exp}(g)\rangle \\
& =\left\langle A_{\nu}^{-}(q \cdot 1) \operatorname{Exp}(f), \operatorname{Exp}(g)\right\rangle+\left\langle\operatorname{Exp}(f), A_{\nu}^{-}(q \cdot 1) \operatorname{Exp}(g)\right\rangle \\
& +\left\langle\operatorname{Exp}(f), A_{\nu}^{-}(q f) \operatorname{Exp}(g)\right\rangle \\
& =\left\langle A_{\nu}^{-}(q \cdot 1) \operatorname{Exp}(f), \operatorname{Exp}(g)\right\rangle+\left\langle A_{\nu}^{+}(q \cdot 1) \operatorname{Exp}(f), \operatorname{Exp}(g)\right\rangle \\
& +\left\langle\Lambda_{\nu}(q) \operatorname{Exp}(f), \operatorname{Exp}(g)\right\rangle \\
& =\left\langle\left(A_{\nu}^{-}(q \cdot 1)+A_{\nu}^{+}(q \cdot 1)+\Lambda_{\nu}(q)\right) \operatorname{Exp}(f), \operatorname{Exp}(g)\right\rangle .
\end{aligned}
$$

This gives the statement.

1. L. Accardi and A.Boukas, Random variables and positive definite kernels associated with the Schroedinger algebra, Proceedings of the VIII International Workshop Lie Theory and its Applications in Physics, Varna, Bulgaria, June 16-21, 2009, pages 126-137, American Institute of Physics, AIP Conference Proceedings 1243.

2. L. Accardi, A. Boukas and J. Misiewicz, Existence of the Fock Representation for Current Algebras of the Galilei Algebra, Quantum Probability and Related Topics XXVII. Proceedings of the 30th Conference Santiago, Chile, 23-28 November (2009) 1-33.

3. L. Accardi and A. Boukas, The Centrally Extended Heisenberg Algebra and Its Connection with Schrodinger, Galilei and Renormalized Higher Powers of Quantum White 
Noise Lie Algebra, American Institute of Physics Conference Proceedings 1243 (2009), 115-125.

4. L. Accardi and M. Bożejko, Interacting Fock space and Gaussianization of probability measures, Infin. Dimens. Anal. Quantum Probab. Relat. Topics 1 (1998), 663-670.

5. L. Accardi, A. Barhoumi and A. Riahi, White Noise Lévy-Meixner Processes Through a Transfer Principal From one-Mode to one-Mode Type Interacting Fock, Infin. Dimensional Anal. Quantum Prob. Related Topics 13 (2010), 435-460.

6. L. Accardi, Y.G. Lu and I. Volovich, Interacting Fock Spaces and Hilber Module Extensions of the Heisenberg Commutation Relations, Publications of IIAS, Kyoto, 1997.

7. L. Accardi, Y.G. Lu and I. Volovich: White noise approach to classical and quantum stochastic calculi, Lecture Notes of the Volterra International School of the same title, Trento, Italy (1999), Volterra Center preprint 375, Università di Roma Tor Vergata.

8. L. Accardi, Y.G. Lu and I. Volovich, Quantum Theory And Its Stochastic Limit, Springer-Verlag Berlin and Heidelberg Gmbh \& Co. Kg (Germany), 2002.

9. S. Albeverio, Yu. L. Daletsky, Yu.G. Kondratiev and L. Streit, Non-Gaussian infinite dimensional analysis, J. Funct. Anal. 138 (1996), 1-42.

10. S. Albeverio, Yu.G. Kondratiev and J.L. Silva, How to generalize white noise analysis to non-Gaussian spaces. In. Dynamics of complex and irregular systems (Ph. Blanchard, L. Streit, M. Sirugue-Collin and D. Testard, eds.), World Scientific, Singapore, (1993), 120-130.

11. N. Asai, I. Kubo and H.-H. Kuo, Multiplicative Renormalization and Generating Functions II., Taiwanese Journal of Mathematics, Vol. 8, No. 4 (2004), 583-628.

12. L. Accardi, H. Rebei and H., Ouerdiane, Lévy processes through time schift on oscillator Weyl algebra, Comminications on stochastic analysis, Vol. 6, No. 1 (2012), 125-155.

13. Yu.M. Berezansky and V.D. Koshmanenko, An asymptotic field theory in terms of operator Jacobian matrices, Soviet Physics Dokl. Vol. 14 (1969-1970), 1064-1066.

14. P.J. Feinsilver and R.Schott, Algebraic structures and operator calculus. Volumes I and III, Kluwer, 1993.

15. P.J. Feinsilver, J. Kocik and R. Schott, Representations of the Schroedinger algebra and Appell systems, Fortschr. Phys. 52 (2004), no. 4, 343-359.

16. U. Franz, Representations et processus stochastiques sur les groupes de Lie et sur les groupes quantiques, Memoire de D.E.A., Université Henri Poincaré - Nancy I (1994)

17. A. Guichardet, Symmetric Hilbert spaces and related topics, Lect. Notes Math. 261, Springer, Berlin, 1972.

18. Y.-J. Lee and H.-H. Shih, Donskers delta function of Lévy processes, Acta Appl. Math. 63 (2000) 219-231.

19. Y.-J. Lee and H.-H. Shih, Analysis of generalized Lévy white noise functionals, J. Funct. Anal. 211 (2004), 1-70.

20. G. Ovando, Four dimensional symplectic Lie algebras, Beitrage Algebra Geom. 47 (2006), no. 2, 419-434.

21. K.R. Parthasarathy, An introduction to quantum stochastic calculus, Birkhaüser Verlag, Basel, 1992.

22. K.R. Parthasarathy and K. Schmidt, Positive definite kernels continuous tensor products and central limit theorems of probability theory, Springer Lecture Notes in Mathematics no. 272, 1972.

23. M. Reed and B. Simon, Functional analysis, Methods of modern mathematical physics, Vol. 1, 1980.

24. K.-I. Sato, Lévy Processes and Infinitely Divisible Distributions, Cambridge Stud. Adv. Math., Cambridge Univ. Press, Cambridge, 1999. 